\title{
Making treatment decisions in a void of information
}

\author{
Revisiting the challenges of the beginning of the COVID-19 pandemic, when doctors and patients \\ had to make treatment decisions without the support of scientific evidence, can provide valuable \\ clues on how to prepare for future pandemics.
}

$t$ is early March 2020, and Dr. A is attending a patient admitted to her ward with severe COVID-19. The patient, Mr. X, a 75-year-old male with hypertension and mild obesity, had been admitted to the hospital with bilateral pneumonia and $85 \%$ oxygen saturation while breathing room air. In previous days, more than 100 patients with COVID-19 had been admitted at Dr. A's hospital, among whom 15 needed admission to the intensive care unit and 10 had already died. That same morning, a colleague had presented a literature review on treatment options for COVID-19. The conclusion was that no good-level evidence for the efficacy of any treatment for that condition existed, but some in vitro data and small observational studies (some in preprint form) suggested that some drugs could work. A critical review of those studies indicated a high risk of bias. With that in mind, Dr. A speaks to Mr. X:

Dr. A: "We will provide supportive therapy, including oxygen, to you; unfortunately, no medical treatment has been proven to have any relevant benefit for your condition so far."

Mr. X: "So there is nothing else you can give me?"

Dr. A: "Well, there are some very preliminary and low-quality data on some drugs, but there is a risk of adverse events, and we really don't know whether they work at all."

Mr. X: “It doesn’t matter. I will take the risk."

Scenes like that were the norm at the beginning of the COVID-19 pandemic. In other variations, patients preferred not to receive any drug if it was not clearly effective, and others just said that they would rely on their doctor's decisions. Dr. A finds herself trapped in an ethical dilemma. As a physician, she lives by the 'first, do not harm' and evidence-based medicine principles. Dr. A is an experienced, highly respected physician. Nevertheless, she now hesitates. She has already seen a few patients in a situation similar to that of Mr. X who died. At the very end, she knows those 'potentially effective' drugs well (such as lopinavir/ritonavir, hydroxicloroquine and azithromycin, which will later fail to show any benefit), as she has prescribed them in other conditions, and in general, she does not consider them 'dangerous'. Some colleagues also mention that corticosteroids or other inmunosuppresants might theoretically be useful, as some patients seem to develop an hyperinflammatory state. What if any of these drugs is later shown to be life-saving?

Physicians are frequently confronted with situations for which no strong evidence is available to inform their decisions. Practical wisdom facilitates the process by providing adequate context and appropriately framing the specific situation of the patient. However, in the first stages of the COVID-19 pandemic, there was nothing that could be called 'best evidence' and there was no practical wisdom to rely on. An unfathomable amount of information emerged every day, for which achieving a critical opinion was simply impossible. Encouraging preliminary results could be (and actually were) mistaken as decision-informing data instead of information useful for the design of new studies. But patients were dying. In such situations, the paradigm of evidence-based medicine is seriously challenged, and some behavioral and psychological variables of the physician become prominent: by nature, when confronted with uncertainty, some people prioritize potential benefits, while others are more worried about potential harm.

Is there anything that can be learned from the mistakes made in the treatment of patients with COVID-19? There must be. A new paradigm must be built for making decisions in such situations. First, hospitals should be prepared to perform emergency clinical trials during outbreaks. This is not that utopic. For this, daily activities should be permeated by a culture of clinical research, and organizational structures (ethics committees, support personnel, etc.) should have operating procedures that allow a rapid reaction. Enough staff should be trained in good clinical practices. At the supra-hospital level, networks for clinical research should be formed, if they do not exist already, again with operating procedures that allow a timely reaction for the development of pragmatic research protocols and the organization of large-scale trials. Second, decision-making guidance for such situations should be developed. Clinicians should have as a priority the inclusion of their patients in randomized trials and, where that is not possible, observational cohorts must be organized with follow-up and data collection that allow appropriate analysis of outcomes and adverse events. This would allow the analysis of outcome predictors and would serve as a historical cohort for future comparisons. Third, local protocols for treatment in the absence of national or regional guidelines and supportive evidence should be made by consensus after literature review, with consideration of potential benefits and harm, costs and implementation issues; such protocols might be submitted to the local ethics committee for evaluation, and in all cases, patients should provide consent after being informed.

Even after all this is done, not all doubts in Dr. A's mind will be resolved. However, the decisions that she and Mr. X take will be supported by a procedure that allows evaluation of performance and, in the worst-case scenario, it will at least be useful for the next patients to come.

\section{Jesús Rodríguez-Baño (D) 1,2,3凶}

${ }^{1}$ Unidad Clínica de Enfermedades Infecciosas y Microbiología, Hospital Universitario Virgen Macarena, Seville, Spain. ${ }^{2}$ Departamento de Medicina, Universidad de Sevilla, Seville, Spain. ${ }^{3}$ Instituto de Biomedicina de Sevilla, Seville, Spain. $\bigotimes_{e-m a i l: j e s u s r b @ u s . e s}$

Published online: 22 March 2021

https://doi.org/10.1038/s41591-021-01293-x

Competing interests

The author declares no competing interests. 